\title{
AUTOMATIC DETECTION AND EXTRACTION ALGORITHM OF INTER-GRANULAR BRIGHT POINTS
}

\author{
Song Feng ${ }^{1,2}$, Kai-fan Ji ${ }^{1}$, Hui Deng ${ }^{1}$, Feng Wang ${ }^{1}$, and XiaO-dong Fu ${ }^{1}$ \\ ${ }^{1}$ Computer Technology Application Key Lab of Yunnan Province and Faculty of Information Engineering and \\ Automation, Kunming University of Science and Technology, Kunming 650500, China \\ E-mail:fs@cnlab.net \\ ${ }^{2}$ Key Laboratory of Solar Activity, National Astronomical Observatories, Chinese Academy of Sciences,
} Beijing 100012, China

(Received August 31, 2012; Revised November 10, 2012; Accepted November 15, 2012)

\begin{abstract}
Inter-granular Bright Points (igBPs) are small-scale objects in the Solar photosphere which can be seen within dark inter-granular lanes. We present a new algorithm to automatically detect and extract igBPs. Laplacian and Morphological Dilation (LMD) technique is employed by the algorithm. It involves three basic processing steps: (1) obtaining candidate "seed" regions by Laplacian; (2) determining the boundary and size of igBPs by morphological dilation; (3) discarding brighter granules by a probability criterion. For validating our algorithm, we used the observed samples of the Dutch Open Telescope (DOT), collected on April 12, 2007. They contain 180 high-resolution images, and each has a $85 \times 68 \operatorname{arcsec}^{2}$ field of view $(\mathrm{FOV})$. Two important results are obtained: first, the identified rate of igBPs reaches $95 \%$ and is higher than previous results; second, the diameter distribution is $220 \pm 25$ $\mathrm{km}$, which is fully consistent with previously published data. We conclude that the presented algorithm can detect and extract igBPs automatically and effectively.
\end{abstract}

Key words : techniques: image processing — Sun: granulation — Sun: photosphere

\section{INTRODUCTION}

Granulation is the dominant feature on the Solar surface. The dark inter-granular lanes are formed as a result of plasma flows and are regions where intergranular Bright Points (igBPs) usually can be found (Dunn \& Zirker 1973; Spruit 1979; Title et al. 1987). The significance of igBPs on the atmospheric transport and dynamics led to several studies on their distributions such as area coverage, number and intensity. These parameters give us a better understanding of the small-scale photospheric feature. In order to detect and study igBPs, several algorithms for automatic detection have been developed (Berger et al. 1995; Bowelet \& Wiehr 2001, 2003, 2007; Utz et al. 2009; Crockett et al. 2009, 2010).

Berger et al. (1995) utilized a modified "blob finding" algorithm to extract igBPs from inter-granular lanes. For enhancing igBPs, the difference between $3 \times 3$ square neighborhood and $7 \times 7$ is calculated in an image. The "blob enhanced" image is processed with a threshold operation to obtain a binary image, which is unity at the locations of igBPs (and at some residual granulation peaks) and zero elsewhere. One copy of the binary image is dilated and eroded with a 5 pixel "+" kernel to eliminate most of the large residual granulation noises, and to separate proximal igBPs. A second

Corresponding Author: K.-F. Ji copy of the binary image is smoothed by the median gray-level value in the $3 \times 3$ pixels neighborhood. The two processed images are then added, using a Boolean "OR" operation to create the binary igBPs map.

A multiple-level tracking (MLT_4) has been presented by Bovelet \& Wiehr (2001, 2003, 2007). Their procedure uses the intensity to identify igBPs via a four-stage process. First, an equidistant intensity level is given by an intensity step width from maximum to zero intensity on the Solar surface, producing a pattern of cells surrounding each local intensity maximum. Second, all the pixels within these cells are normalized from 0 to 100 according to their intensities. Next, the cells are shrunk to reasonable sizes, resulting in a single cut-off threshold to their normalized intensity profiles. Finally, adjacent features at an appropriate reference level are merged by removing the respective common separation lines.

Utz et al. (2009) developed an updated version of the MLT_4 algorithm (Bovelet \& Wiehr 2007) and then applied it to the Hinode/SOT observed data sets. Their algorithm consists of three major steps: segmentation, clean-up and identification. The segmentation is based on the idea of a region-growing algorithm, following the border of a feature from the brightest pixels to the faintest ones. The brightest pixel of an image is identified and then used to initialize the first feature. In the next step, pixels of slightly lower brightness are 
obtained and merged into the first feature if they are adjacent. Otherwise, they are used to initialize the next feature. Iterating these steps, the image is segmented into single features. In the clean-up step, a threshold value is applied to clean up several brighter granules. In the final step, the size on each inter-granular bright point (igBP) is extracted. The sizes are determined by a brightness threshold set to be $30 \%$. All the pixels in the segmented section are considered, from the brightest pixel till those with brightness less $30 \%$ than the brightest one.

A compass search method has been used by Crockett et al. (2009). First, the original image is split into $128 \times 128$ pixel $^{2}$ areas and then the intensity threshold is determined by the values of the mean intensity minus $0.8 \times$ the sigma value of each split image. All the structures under this threshold are considered as lanes and not investigated by their algorithm. Next, the large structures are removed, if the pixels of a bright region will be greater than 7 pixels in all the directions of the compass. At last, the identified igBPs are grown to recover pixels that could have been removed in the first stage of this process. An updated algorithm of the compass search has been developed by Crockett et al. (2010). The significant improvement is that the original image is no longer split and a newly growing threshold is provided according to each object's center of gravity.

The aim of the paper is to present a simple and concise method for identifying igBPs from a large volume of observed data. The paper is organized as follows. The observations are described in Section 2, and the automatic algorithm is detailed in Section 3. Finally, our result and discussion are given in Section 4, and Section 5 summarizes our conclusions.

\section{OBSERVATIONS AND DATA SET}

The data set used in this study was obtained with the Dutch Open Telescope (DOT) on April 12, 2007. The telescope has an optical Solar telescope with a main mirror of 45 centimeters. The spatial resolution of reconstructed DOT images may reach 0.2 arcsec. The data set has a field of view $(\mathrm{FOV})$ of $85 \times 68 \operatorname{arcsec}^{2}$ with a spatial sampling of $0.071 \mathrm{arcsec} / \mathrm{pixel}$ (about $51.5 \mathrm{~km}$ on the Sun). The complete time series consist of 180 G-band images. The time lapse between successive images is 0.5 minute. An image is shown in Fig. 1, where multiple igBPs may be detected. The data set can be downloaded from the web site*

\section{TECHNIQUE FOR AUTOMATIC DETEC- TION AND EXTRACTION}

The largest uncertainty in detecting and extracting igBPs with an automatic algorithm is due to their small

\footnotetext{
*ftp://dotdb.strw.leidenuniv.nl/2007/2007-04-12/QuietSun/ FITS/GBand/
}

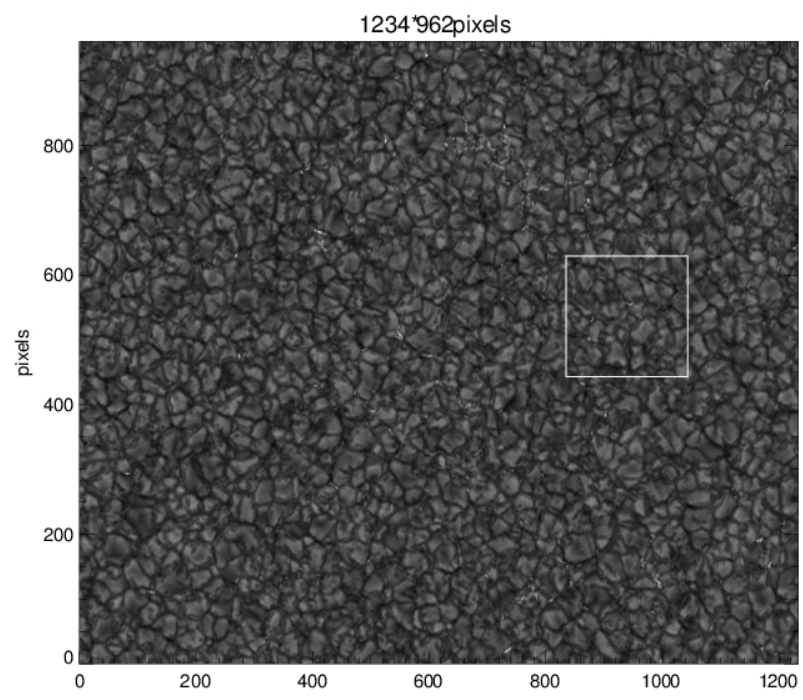

Fig. 1.- A $85 \times 68 \operatorname{arcsec}^{2}$ high spatial resolution (51.5 $\mathrm{km} /$ pixel) image of Solar granulation from the DOT. A large number of igBPs can be seen in the image. They retain a high intensity compared to the photospheric background and are located within the inter-granular lanes. The white lines indicate a subsection enlarged in Figs. 2 and 4.

scale and fuzzy boundary. Three major characteristics of igBPs have been utilized by most automatic algorithms: bright, size and gradient. For example, they have brighter intensity than the surrounding objects; their sizes are small, and they possess a larger gradient. However, the processing algorithms have some limitations. If several igBPs are merged to form a ribbon structure, the size threshold with small-scale can cause them to be discarded. If the intensity is used as a criterion, a brighter feature on top of granules would also be extracted as an igBP, and dull igBPs could be lost under the same criterion. Intensity gradient is usually used, but the difficulty with this approach is that in practice the igBP-profile is usually not known. Furthermore, the profile often varies heavily along different observations. A single characteristic is insufficient to ensure that the detection results are accurate and correct. Therefore, an automatic algorithm should use all the previous characteristics to detect and extract igBPs.

There are two difficulties in an automatic detection algorithm for igBPs: (1) how to correctly disentangle igBPs from the inter-granular lanes; (2) how to appropriately define igBP sizes. Our algorithm uses three major processing steps to solve the problem:

(1) obtaining the candidate "seed" regions with Laplacian;

(2) determining the boundary and size of igBPs with morphological dilation;

(3) discarding brighter granules with a probability criterion.

The processing steps are validated by a DOT ob- 
served image series (e.g., Fig. 1 is one of the series). Fig. 2a is a $150 \times 150$ pixel $^{2}$ box, highlighted in Fig. 1. In order to better demonstrate the algorithm, the following procedures will be performed on the image series.

\subsection{Obtaining the Candidate "Seed" Regions with Laplacian}

An igBP is usually the brightest features and an isolated point in the G-band filtergram. The most common way to look for isolate points is to run a Laplacian mask (Eq. 1) in the image. The following equation is an extended Laplacian operation.

$$
W=\left[\begin{array}{ccc}
-1 & -1 & -1 \\
-1 & 8 & -1 \\
-1 & -1 & -1
\end{array}\right]
$$

This procedure involves computing the sum of the products of the coefficients with gray levels contained in the region encompassed by the mask. The response of the mask at any point in the image is given by

$$
R=w_{1} z_{1}+w_{2} z_{2}+\ldots+w_{9} z_{9}=\sum_{i=1}^{9} w_{i} z_{i}
$$

where $w_{i}$ is the value corresponding to each location in Eq. 1, and $z_{i}$ the intensity of the pixel associated with the mask coefficient $w_{i}$. The response of the mask is defined with respect to its center location. An isolate point is detected where the mask is centered if $R \geq T$, where $T$ is a nonnegative threshold and $R$ is given by Eq. 2. This expression measures the weighted differences between the central point and its neighbors. An igBP is quite different from its surroundings, and it is easily detected by the mask (Eq. 1).

We illustrate the detection of isolated points from an image. Since pixel noises on the image can be misinterpreted by an automated algorithm, an average smoothing with a sliding window of $3 \times 3$ is utilized to eliminate pixel noises. Next, the smoothed image is calculated by Eq. 2, and Fig. 2b shows the result to the sample (Fig. 2a). To facilitate the illustration, the image obtained by Eq. 2 is called "Laplacian image" (e.g., Fig. 2b) in this paper. In the "Laplacian image", a positive response indicates that the intensity value of the coordinate position is higher than its 8 adjacent pixels, and the opposite for a negative response. In our algorithm, the positive mask response is regarded as a brighter region (e.g., an igBP or a brighter granule), and the negative response is regarded as a dark region (e.g., an inter-granular lane).

A candidate is a "seed" region where an igBP could be found. In order to select the "seed" regions, a threshold $T$ will be chosen. In this process, the threshold criterion is set from the result of the mean image plus three times the standard deviation $(\mu+3 \sigma)$. When the procedure is finished, the original image has
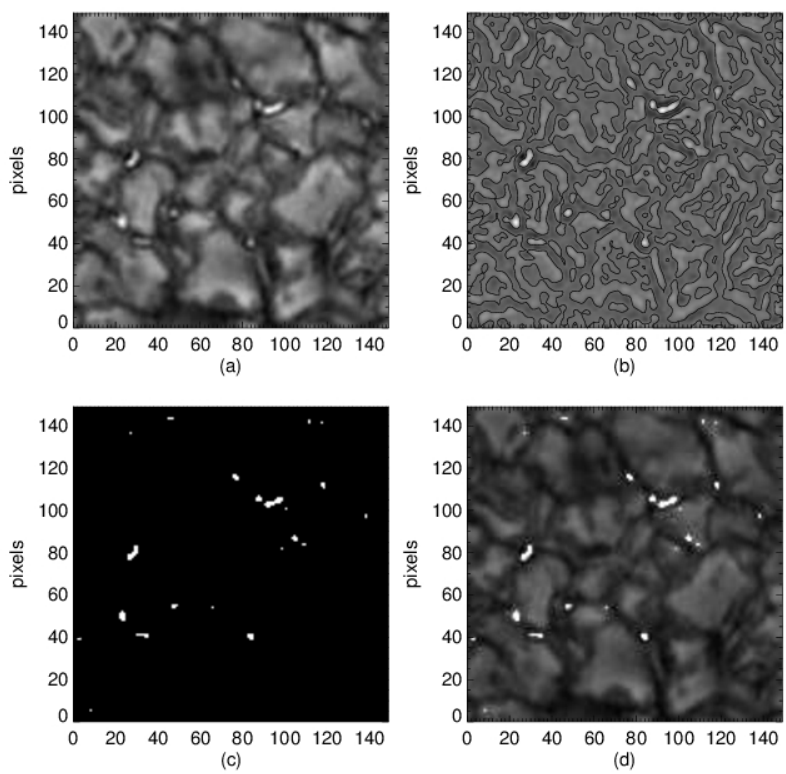

Fig. 2.- (a) The highlighted $150 \times 150$ pixel $^{2}$ box in Fig. 1. (b) The same image after application of Eq. 2. (c) The binary image of candidates is obtained by setting a threshold $(\mu+3 \sigma)$. (d) Positions marked in white represent candidates in Fig. 2a. The candidates contain several brighter granules in Fig. 2d.

been transformed into a binary one (Fig. 2c). The "white" regions of Fig. 2c are obtained by the threshold $T$. Since igBPs are restricted inside the inter-granular lane, their diameters are determined by the width of inter-granular lanes. Because the candidates contain only those pixels of highest gradient, their sizes are less than real sizes of igBPs in practice. To facilitate the observation, Fig. 2c is enlarged and the region is shown partly in Fig. 3. It can be seen that the "white" candidates do not reach the boundary of the lane. The reason why the size of a candidate is less than the one of a real igBP is that some pixels with lower gradient cannot attain the threshold $(\mu+3 \sigma)$ and are discarded. Another limitation is that granular features (e.g., brighter granules) could be regarded as candidates, if they possess several brightness maxima. The superposition of Figs. 2a and 2c is displayed in Fig. 2d, where several candidates can be seen on top of the granules. These negative impacts could be minimized by the following steps.

\subsection{Determining the Boundary and Size of igBPs with Morphological Dilation}

In this step, a morphological dilation operator is used to determine the sizes of these candidates. The morphology is based on set theory. Sets in mathematical morphology represent objects in an image. A binary image is a complete morphological description of an image in $2 \mathrm{D}$ integer space $\left(Z^{2}\right)$, where elements are either 


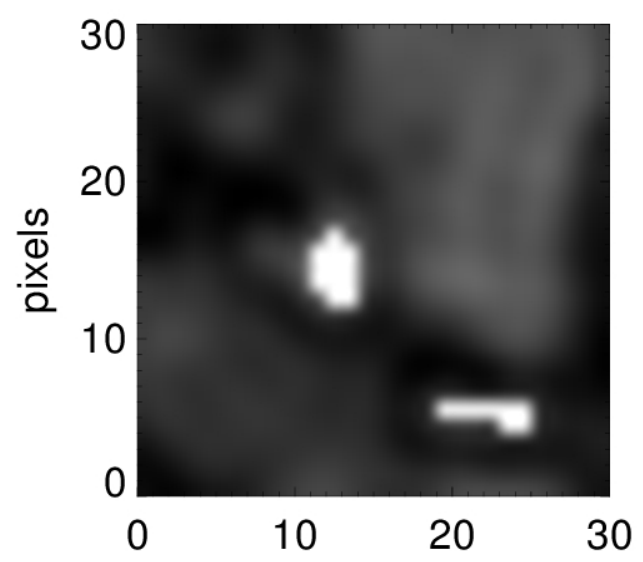

Fig. 3.- Enlargement of Fig. 2d is shown partly to facilitate the explanation. It can be seen from the map that the two brightest sizes do not extend till the boundary of inter-granular lanes.

"0" or " 1 ". A structuring element of the morphological dilation operator is defined as

$$
B=\left[\begin{array}{lll}
1 & 1 & 1 \\
1 & 1 & 1 \\
1 & 1 & 1
\end{array}\right]
$$

Before determining the sizes of igBPs, the pixels surrounding each candidate and with slightly lower gradient than adjacent candidates should be recovered and regarded as sizes of igBPs. For recovering the pixels, each candidate should be expanded. The $3 \times 3$ structuring element (Eq. 3) enlarges a boundary having a thick pixel. The values associated with all boundary pixels would be derived from a "Laplacian image".

On the "Laplacian image", those negative response imply that the intensity levels at their coordinate positions are lower than their adjacent space. They are then considered to be inter-granular lanes. Pixels with negative response are removed from the boundaries, which are enlarged by morphological dilation (Eq. 3). The remaining pixels are immediately merged into adjacent candidates and contained by the final detection (Fig. 4a).

Repeating the previous procedure, each candidate region is enlarged by its adjacent space again, and the result is shown in Fig. 4b. Fig. 4c is the difference between Fig. 4b and Fig. 2c. It can be seen from Fig. $4 \mathrm{c}$ that not all the 8 adjacent pixels for each candidate are merged by the final detection. Since the diameter of igBPs is most likely limited by the width of the intergranular lane, their diameter cannot be larger than the characteristic width of the inter-granular lanes, which varies in the range of $100-400 \mathrm{~km}$ (Wiehr \& Bovelet 2004; Utz et al. 2009; Abramenko et al. 2010). Because the identified data set is a spatial sampling of 0.071 arcsec (about $51.5 \mathrm{~km}$ on the Sun), each candi-
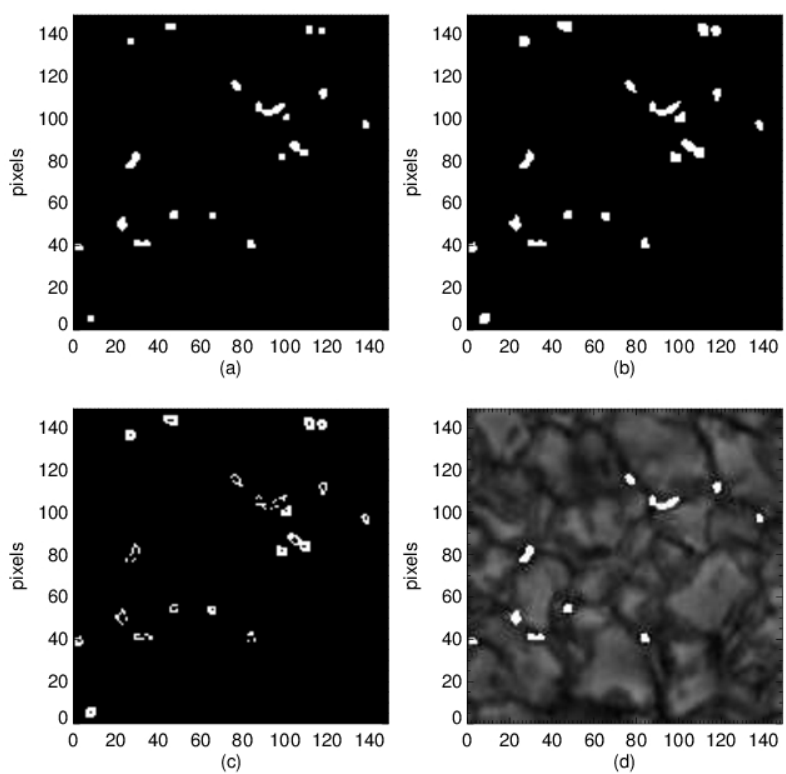

Fig. 4.- (a) Fig. 2c dilated by a morphological operator. (b) Fig. 4a dilated by a morphological operator. (c) A binary image displays the distinction between a candidate (Fig. 2c) before and after double dilation. Not all of the 8 adjacent pixels for each candidate are recovered. (d) The final detection result marked in white. The map demonstrates the high degree of accuracy in the reconstruction.

date is enlarged twice by Eq. 3. If the candidate is a real igBP, it should be extended to the boundary of inter-granular lane. At the end of this step, igBP sizes are determined.

\subsection{Discarding Brighter Granules with a Prob- ability Criterion}

When the boundary of each igBP is determined thanks to the previous step, a probability threshold can be utilized to eliminate those brighter regions within granules. There are two major distinctions between igBPs and brighter granules. One is that igBPs usually reside within inter-granular lanes, whereas a brighter granule is located on top of a granulation structure. The other is that an igBP is an isolated point, whereas a brighter granule is a much larger region. When the previous procedure of "seed" expanding is finished, the boundaries of igBPs have been expanded to intergranular lanes while brighter granules have not. By applying a probability threshold, a ratio of each candidate is calculated, respectively. When the second morphological dilation is executed, the number of expanded pixels associated with negative measurement in the "Laplacian image" is denoted by $M$, the total number of expanded pixels is denoted by $N$, and their ratio is given by $R=M / N$. If the candidate is an isolate igBP fully surrounded by a lane, $\mathrm{M}$ will be equal to $\mathrm{N}$, and so $R=1$. If it is a brighter granular, the rate will 


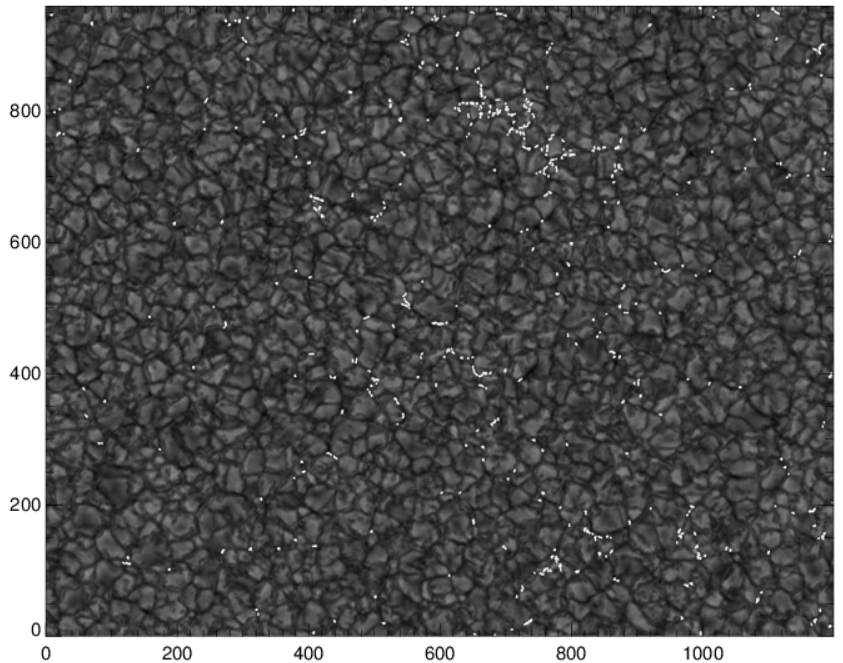

Fig. 5.- After applying the three-step process described in Section 3, the map displays the resulting binary image projected onto Fig. 1. Regions marked in white represent detected BP positions.

be less than 1 . Therefore, the ratio indicates the probability of being an igBP. If $R$ is less than a predefined threshold, the candidate is most probably an element on top of a granular feature (i.e., a brighter granule) and is discarded by our algorithm. The threshold is set to be 0.7 , in our case.

The brightest elements in Fig. 4d are the final result of igBPs. Fig. 4d demonstrates that igBPs have been detected and extracted with a high degree of accuracy. Finally, after applying the three processes described above, the original image is marked as binary detection frame of the entire field of view, and the igBPs are indicated at the original location (Fig. 5).

\section{RESULTS AND DISCUSSION}

The intensity of an igBP is from 0.8 to 1.8 times the mean photospheric intensity (Sánchez Almeida et al. 2004) and its shape is close to a circular structure with average eccentricity of 1.5 (Berger et al. 1995). The criterion is utilized to judge an igBP extracted. All the objects are considered as igBPs under the criterion.

\subsection{Identified Rate and Undetected Analysis of igBPs}

In order to validate the correctness of the detection and extraction, 10 samples were randomly selected. The igBPs were primarily identified visually using a stringent constraint to assure that the identified object was an igBP. Our algorithm was applied to the sample, and the numbers of correct, incorrect and undetected features were recorded. A total of 1350 igBPs were visually identified in this examination. A total of 1292 features are extracted by the algorithm presented, involving 1280 correct features and 12 incorrect. The results lead to a $95 \%$ identified rate with $5 \%$ of undetected rate, and $0.9 \%$ incorrect rate. Crockett et al. (2009) utilized the "Compass Search" algorithm to extract igBPs. They employed the observations data with the Swedish Solar Telescope (SST) and revealed a $90 \%$ identified rate of igBPs. Our result is slightly higher than their result. As the experimental result is derived by a comparison through visual recognition, the comparison might lead to some inaccuracy.

Several regions were identified incorrectly by the algorithm: this is because brighter granules are close to the inter-granular lanes. A region is then identified by the enlarged procedure as a possible igBP structure surrounded entirely by lanes. Exploding granules may be another reason of incorrect detections.

In some cases, a few igBPs could be undetected. If the igBP is the later period of evolution, its intensity gradient cannot attain the required threshold level $(\mu+$ $3 \sigma$ ) and can be discarded.

\subsection{Distribution of igBP Diameters}

Assuming an igBP to have circular geometry, the equivalent diameter of the igBP is calculated by its sizes. In order to examine the accuracy of the defined size, the diameter distribution is obtained as follows. Diameters around and below the theoretical resolution limit (in our case below 3 pixels) have to be discarded because these regions normally could never be resolved. The diameter distribution incorporates a total of 18380 size measurements in $180 \mathrm{G}$-band images; it follows a Normal distribution with fit parameters $\sigma=25$ $\mathrm{km}$ (standard deviation) and $\mu=220 \mathrm{~km}$ (mean value). Fig. 6 displays the diameter distribution. The result agrees with that of Bovelet \& Wiehr (2003) perfectly. Their result was based on data from the DOT taken on April 1, 2001. They derived most frequent diameter of $220 \pm 25 \mathrm{~km}$ as well. The peaks of the igBP distribution are higher than the DOT telescope diffraction limit.

\subsection{Analysis and Discussion}

In our algorithm there are two parameters: the threshold $T(\mu+3 \sigma)$ and the probability threshold (0.7), which may be adjusted. To test the influence of $T$, the result of $3 \sigma$ was compared with those of $2 \sigma$ and $4 \sigma$ using Fig. 1, where 135 igBP features are found visibly. Under the same criteria, the result of the automatic recognition is shown in Table 1.

The results of Table 1 imply that if a small threshold is selected, the correct number is improved, the undetected number is decreased, but the incorrect number is increased. If a large threshold is used, the incorrect number is decreased, however, not only the correct number is decreased, but also the undetected number 


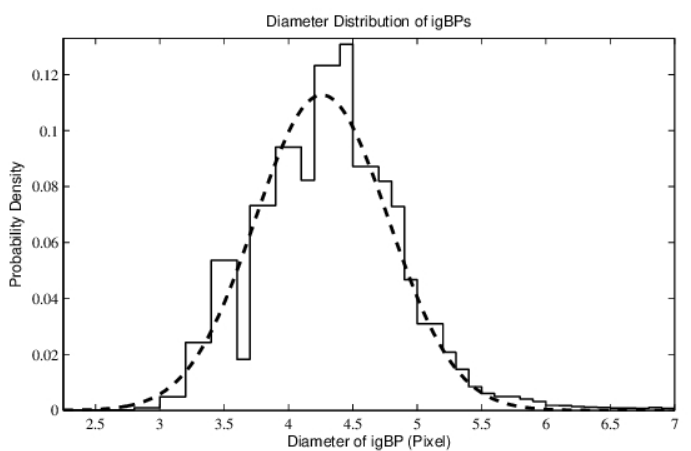

Fig. 6.- Distribution of igBP sizes (histogram), fitted with a Normal distribution (dashed line). The fitted parameter is $4.2 \pm 0.5$ pixel-widths (about $220 \pm 25 \mathrm{~km}$ on the Sun).

Table 1.

Experimental results for the threshold T

\begin{tabular}{ccccc}
\hline \hline Values of T & Total & Correct & Incorrect & Undetected \\
\hline $2 \sigma$ & 214 & 133 & 81 & 2 \\
$3 \sigma$ & 132 & 128 & 4 & 7 \\
$4 \sigma$ & 77 & 75 & 2 & 60 \\
\hline
\end{tabular}

is increased. In order to ensure the algorithm accuracy and correctness, we select the threshold of $3 \sigma$ with our algorithm.

The probability threshold from 0.5 to 0.9 is investigated using Fig. 1. If the threshold is increased, each igBP detected looks like an isolate point, but the ribbon structures might not be detected. On the other hand, if the threshold is decreased, the ribbon structures cannot be missed, but a few brighter granules might be detected.

\subsection{Algorithm Efficiency}

The experiments were carried out on a laptop equipped with $2.27 \mathrm{GHz} \mathrm{CPU}$ and $2 \mathrm{G}$ RAM. The algorithm is implemented in the IDL environment. It takes about 43 seconds to detect all the igBPs in a $1200 \times 960$ pixels $^{2}$. Therefore, it is acceptable to handle vast observed data set rapidly.

\section{CONCLUSIONS}

In this paper we presented a new automatic algorithm, employing Laplacian and Morphological Dilation (LMD), to detect and extract igBPs in the Solar photosphere. First, we utilized a Laplacian operation to obtain candidates. Then the candidates were expanded by morphological dilation to acquire the bound- ary of igBPs. Finally, a probability criterion was used to discard brighter granules. Based on the positions of igBPs obtained by the algorithm, other features such as center of gravity, size, diameter, eccentricity, and mean intensity, can be calculated automatically in each field of view. Since only two parameters need to be adjusted, it is easy to use the algorithm to investigate the characteristics of igBPs. The recognition rate of the algorithm is higher and results agree well with earlier published values, implying that the automatic algorithm is entirely feasible to acquire the statistical parameters. The algorithm is concise and efficient, indicating that it can deal with a large volume of observed data. Further work will be carried out to develop automatic tracking, in order to obtain more realistic dynamical characteristics. Finally, we emphasize that the presented algorithm could be applied to some high-contrast point-like features such as granules, BPs observed with different band filtergrams, small and large-scale magnetic fluxes observed in magnetograms, as well as igBPs.

\section{ACKNOWLEDGMENTS}

The authors thank the anonymous referee for her/his careful reading of the manuscript and constructive comments that improved the original version. This work was supported by the National Natural Science Foundation of China (No: 11163004, 11263004) and the Open Research Program of Key Laboratory of Solar Activity of Chinese Academy of Sciences (No: KLSA201205). The samples used in the present paper are kindly provided by the Dutch Open Telescope (DOT); the authors would like to express their deep gratitude to the DOT staff.

\section{REFERENCES}

Abramenko, V., Yurchyshyn, V., Goode, P., \& Ali, K. 2010, Statistical Distribution of Size and Lifetime of Bright Points Observed with the New Solar Telescope, ApJ, 725, L101

Berger, T. E., Schrijver, C. J., Shine, R. A., Tarbell, T. D., Title, A. M., \& Scharmer, G. 1995, New Observations of Subarcsecond Photospheric Bright Points, ApJ, 454, 531

Bovelet, B., \& Wiehr, E. 2001, A New Algorithm for Pattern Recognition and its Application to Granulation and Limb Faculae, Sol. Phys., 201, 13

Bovelet, B., \& Wiehr, E. 2003, Dynamics of the Solar Active Fegion Finestructure, A\&A, 412, 249

Bovelet, B., \& Wiehr, E. 2007, Multiple-Scale Pattern Recognition Applied to Faint Intergranular G-Band Structures, Sol. Phys., 243, 121

Crockett, P. J., Jess, D. B., Mathioudakis, M., \& Keenan, F. P. 2009, Automated Detection and Tracking of Solar Magnetic Bright Points, MNRAS, 397, 1852 
Crockett, P. J., Mathioudakis, M., Jess, D. B., Shelyag, B., Keenan, F. P., \& Christian, D. J. 2010, The Area Distribution of Solar Magnetic Bright Points, ApJ, 722, L188

Dunn, R. B., \& Zirker, J. B. 1973, The Solar Filigree, Sol. Phys., 33, 281

Sánchez Almeida, J., Márquez, I., Bonet, J. A.,\& Domínguez Cerdeña, I. 2004, Muller, R. Bright Points in the Internetwork Quiet Sun, ApJ, 609, 91

Spruit, H. C. 1979, Convective Collapse of Flux Tubes, Sol. Phys., 61, 363

Title, A. M., Tarbell, T. D., \& Topka, K. P. 1987, On the Relation between Magnetic Field Structures and Granulation, ApJ, 317, 892

Utz, D., Hanslmeier, A., Möstl, C., Muller, R., Veronig, A., \& Muthsam, H. 2009, The Size Distribution of Magnetic Bright Points Derived from Hinode/SOT Observations, A\&A, 498, 289

Wiehr, E., Bovelet, B., \& Hirzberger, J. 2004, Brightness and Size of Small-Scale Solar Magnetic Flux Concentrations, A\&A, 422, L63 